\title{
Genotypic and phenotypic characterization of methicillin-resistant Staphylococcus aureus (MRSA) clones with high-level mupirocin resistance
}

\author{
María González-Domínguez a , Cristina Seral a,b,* ${ }^{\text {a }}$ Carmen Potel ${ }^{c, d}$, Yolanda Sáenz e , Maximiliano Álvarez ${ }^{c, d}$, \\ Carmen Torres e,f , Francisco Javier Castillo a,b \\ a Servicio de Microbiología, Hospital Clínico Universitario Lozano Blesa, San Juan Bosco s/n, 50009, Zaragoza, Spain \\ b Departamento de Microbiología, Facultad de Medicina, Universidad de Zaragoza, C/Domingo Miral s/n, 50009, Zaragoza, Spain \\ c Servicio de Microbiología, Complexo Hospitalario Universitario de Vigo (CHUVI), C/Pizarro, 22, 36204, Vigo Pontevedra, Spain

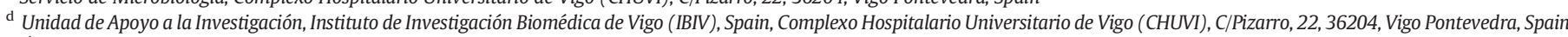 \\ e Área de Microbiología Molecular, Centro de Investigación Biomédica de La Rioja (CIBIR), C/Piqueras, 98, 26006, Logroño La Rioja, Spain \\ ${ }^{\mathrm{f}}$ Área de Bioquímica y Biología Molecular, Universidad de La Rioja, Avda/Madre de Dios, 51, 26006, Logroño La Rioja, Spain
}

\section{A R T I C L E I N F O}

\section{Article history:}

Received 8 November 2015

Received in revised form 24 February 2016

Accepted 25 February 2016

Available online 28 February 2016

\section{Keywords:}

High-level mupirocin resistance

MRSA

Clonal lineages

Resistance genes

\begin{abstract}
A B S T R A C T
A high proportion of methicillin-resistant Staphylococcus aureus isolates recovered in one year period showed high-level mupirocin-resistance (HLMUPR-MRSA) in our environment (27.2\%). HLMUPR-MRSA isolates were mainly collected from skin and soft tissue samples, and diabetes was the main related comorbidity condition. These isolates were more frequently found in vascular surgery. HLMUPR-MRSA was more resistant to aminoglycosides than mupirocin-susceptible MRSA, linked to the presence of bifunctional and/or nucleotidyltransferase enzymes with/without macrolide resistance associated with the $m s r(\mathrm{~A})$ gene. Most of HLMUPR-MRSA isolates belonged to ST125/t067. Nine IS257-ileS2 amplification patterns (p3 was the most frequent) were observed in HLMUPR-MRSA isolates, suggesting the presence of several mupirocin-resistance-carrying plasmids in our environment and promoting the emergence of mupirocin resistance. The presence of the same IS257-ileS2 amplification pattern p3 in 65\% of HLMUPR-MRSA, all of them ST125/t067, suggests a clonal spread in our hospital and community environment which could explain the high prevalence of HLMUPR-MRSA during the study period. An outbreak situation or an increase in mupirocin consumption was not observed.
\end{abstract}

(c) 2016 Elsevier Inc. All rights reserved.

\section{Introduction}

Mupirocin, also known as pseudomonic acid A, is a topical antibiotic that was originally isolated from Pseudomonas fluorescens. It is used for decolonization of nasal carriers of methicillin-resistant Staphylococcus aureus (MRSA) (Sutherland et al., 1985). The increased use of this antibiotic has been accompanied by outbreaks of mupirocin-resistant MRSA (Schmitz and Jones, 1997; Simor et al., 2007).

Mupirocin is an analogue of isoleucine that inhibits protein synthesis by competitively binding to the enzyme isoleucyl-tRNA synthetase (Yanagisawa et al., 1994). The high-level mupirocin-resistant isolates show a MIC greater than $512 \mathrm{mg} / \mathrm{L}$. This resistance is mediated by the acquisition of a plasmid containing the ileS2 gene that encodes an alternative isoleucyl-tRNA synthetase enzyme and is generally flanked by copies of the insertion sequence IS257 (Pérez-Roth et al., 2010; Woodford et al., 1998).

\footnotetext{
* Corresponding author. Tel.: +34-976-556400x4319.

E-mail addresses: cseral@unizar.es, cristiseral@gmail.com (C. Seral).
}

The objective of the study was to identify the prevalence, clonal lineages, resistance mechanisms and virulence genes of high-level mupirocin-resistant MRSA (HLMUPR-MRSA) isolates recovered from inpatients and outpatients in our institution during one year.

\section{Material and methods}

One hundred forty-seven MRSA isolates were collected from clinical samples in the University Teaching Hospital "Lozano Blesa" (Zaragoza, Spain) from July 2009 to July 2010 . Only one isolate per patient was included. The study was conducted retrospectively. Clinical records of all patients were reviewed. For each patient the following data were collected: gender, age, medical service, source of the culture sample and comorbid conditions during the year prior to MRSA isolation (dialysis, diabetes, vascular disease, malignancy, hospitalization, MRSA isolation, medical devices, contact with healthcare settings and surgery). To establish the possible community origin of MRSA isolates, CDC criteria were followed, considering in this category the isolates from outpatients with no hospitalization history during the last year, or from patients within their first $48 \mathrm{~h}$ after admission, who presented no other 
established risk factors for MRSA infection, such as a sojourn in a longterm care facility, dialysis, surgery, indwelling permanent catheters, or medical devices. Antibiotic consumption in the year prior to isolation was studied only in patients who carried HLMUPR-MRSA. Mupirocin consumption data in our hospital were obtained from pharmacy records.

Identification and susceptibility testing was carried out by WIDER $® I$ System (Francisco Soria-Melguizo, Madrid, Spain) and disk diffusion method and interpreted according to the CLSI guidelines (Clinical and Laboratory Standards Institute (CLSI), 2014). High mupirocin MICs (mupirocin MICs $\geq 256 \mathrm{mg} / \mathrm{L}$ ) were confirmed by E-test strip method.

The main genes that encode resistance to macrolides, lincosamide, streptogramin type $B$, aminoglycosides, tetracycline and mupirocin were investigated by PCR (Aarestrup et al., 2000; Aktas et al., 2007; Choi et al., 2003; Larsen et al., 2008; Lina et al., 1999; Udo et al., 2001). Clinical strains usually harbor a complex mixture of resistance plasmids, and laborious time consuming methodologies are required to associate HLMUPR-MRSA with a plasmid type (Leski et al., 1999; Pérez-Roth et al., 2006). To enable the monitoring of HLMUP resistance dissemination in staphylococci, we used a rapid typing method for strain characterization based on the heterogeneous IS257-ileS2 spacer regions (amplification of up- and down- stream IS257-ileS2 spacer regions) found on ileS2encoding plasmids. Four PCR reactions were performed, as previously described (Pérez-Roth et al., 2011), on all mupirocin-resistant isolates.

All isolates were typed by spa typing (Larsen et al., 2008). Only HLMUPR-MRSA isolates were typed by SCCmec, agr typing, PFGE using Smal and MLST as previously described (González-Domínguez et al., 2012). PFGE profiles were analyzed with GelCompar II ${ }^{\circledR}$ software (Applied Maths, Kortrijk, Belgium). Dendrograms were generated by the unweighted pair-group method using arithmetic averages, based on the Dice similarity coefficient with a $1.0 \%$ band position tolerance. PFGE patterns were assigned into pulsotypes (named with capital letters) and subtypes (named with numbers in subscript). Different pulsotypes were considered if the similarity coefficient was $<80 \%$. Different subtypes were considered when the similarity coefficient oscillates in the 80-95\% interval. HLMUPR-MRSA isolates were screened for virulence genes encoding Panton-Valentine leukocidin (PVL) (lukS-PV and lukF-PV genes), exfoliative toxins ETA and ETB (eta and etb genes) and toxic shock syndrome toxin (TSST) (tst gene) (Jarraud et al., 2002; Larsen et al., 2008).

Statistical significance was calculated for comparison of proportions using the chi-square test with Yates' correction. $P \leq 0.05$ was considered statistically significant (SPSS V15.0; SPSS, Chicago, IL).

\section{Results and discussion}

During the course of this study, MRSA prevalence in our hospital was $30.9 \%$, consistent with other resistance rates found in different Spanish hospitals (Cuevas et al., 2008; González-Domínguez et al., 2015; Lozano et al., 2013). Forty MRSA isolates showed HLMUPR, representing $27.2 \%$ of the studied isolates (mupirocin MICs $\geq 256 \mathrm{mg} / \mathrm{L}$ ). This percentage is higher than that found in other Spanish hospital (Daskalaki et al., 2009). Other isolates were mupirocin-susceptible (i.e. MIC, $<8 \mathrm{mg} / \mathrm{L}$ ).

Mupirocin resistance appears to emerge easily in health centers with unrestricted policies that allow widespread use of mupirocin for long periods (Lee et al., 2011). Between 2009 and 2010, mupirocin consumption in our hospital stayed in approximately 1250 units per year (the consumption in the community could not be established). This mupirocin consumption has remained stable over the years. A guideline for surveillance and control of MRSA in hospitals was published in Spain in 2008 (Rodríguez-Baño et al., 2008). Active surveillance cultures to detect asymptomatic MRSA colonization is recommended in all guidelines for control and prevention of MRSA. In general, the guidelines recommends screening the patients at high risk of colonization (previously colonized, patients with multiple hospital or healthcare facilities admissions with high prevalence of MRSA). In our hospital, mupirocin $2 \%$ nasal ointment is used for decolonization of nasal carriers of MRSA after positive MRSA screening.

No statistically significant differences were found between patients with HLMUPR-MRSA and mupirocin-susceptible MRSA isolates, regarding to age (median: 75 years versus 72 years; $P=0.124$ ), male gender (50.0\% versus $59.8 \% ; P=0.285)$, community onset (25.0\% versus $17.7 \% ; P=0.326)$.

In relation to comorbidity conditions associated with patients included in our study, diabetes was more frequently found in patients with HLMUPR-MRSA than mupirocin-susceptible MRSA isolates (35.0\% versus $18.7 \% ; P=0.037$ ); while the presence of malignancy (10.0\% versus $25.2 \% ; P=0.044$ ) or medical devices (12.5\% versus $30.8 \% ; P=0.024)$ were more related with patients who were infected with mupirocin-susceptible MRSA isolates.

HLMUPR-MRSA were more frequently collected from skin and soft tissue samples than mupirocin-susceptible MRSA isolates ( $72.5 \%$ versus 27.1\%; $P<0.001$ ). This association between HLMUPR-MRSA and skin and soft tissue infections has also been described by other authors (Daskalaki et al., 2009). HLMUPR-MRSA was more frequently found in vascular surgery service than mupirocin-susceptible MRSA (30.0\% versus 7.5\%; $P<0.001$ ). No significant differences in the prevalence of other medical services were found. HLMUPR-MRSA were less frequently collected from lower respiratory tract samples than mupirocinsusceptible MRSA isolates ( $2.5 \%$ versus $25.2 \% ; P=0.002$ ).

During the year prior to isolation, the antibiotics most frequently prescribed in patients who carried these HLMUPR-MRSA isolates were beta-lactams (57.5\%) and mupirocin (30\%). These results were not obtained in the group of mupirocin-susceptible MRSA isolates.

An aminoglycoside resistance phenotype was more frequently observed among HLMUPR-MRSA than mupirocin-susceptible MRSA isolates (gentamicin: $85.0 \%$ versus $11.2 \% ; P<0.001$, kanamycin: $97.5 \%$ versus $82.2 \% ; P=0.016$, tobramycin: $92.5 \%$ versus $64.5 \% ; P=0.001$, amikacin: $85.0 \%$ versus $42.0 \% ; P<0.001$, streptomycin: $12.5 \%$ versus $19.6 \% ; P=0.314$, netilmicin: $7.5 \%$ versus $0 \% ; P=0.019$ ) (Fig. 1 ). Aminoglycoside resistance was encoded by aac $\left(6^{\prime}\right)$-le-aph $\left(2^{\prime \prime}\right)$-Ia ( $85.0 \%$ versus $11.2 \% ; P<0.001)$ and $\operatorname{ant}\left(4^{\prime}\right)$-Ia $(85.0 \%$ versus $64.5 \% ; P=0.016)$ genes. A high rate of macrolide resistance was observed, but no significant differences were found between high-level mupirocin-resistant and mupirocin-susceptible isolates (erythromycin: $75.0 \%$ versus 58.8\%; $P=0.07$, azithromycin: $75.0 \%$ versus $58.8 \% ; P=0.07$, spiramycin: $17.5 \%$ versus $13.1 \% ; P=0.496$, clindamycin: $17.5 \%$ versus $12.1 \% ; P=0.4)$. The difference in the prevalence of erythromycin and clindamycin resistance is due to the high percentage of the M phenotype that implicates erythromycin-resistant but clindamycinsusceptible pattern. Macrolide resistance by efflux due to the $\operatorname{msr}(\mathrm{A})$ gene is related with this phenotype. The main macrolide resistance gene found in our HLMUPR-MRSA isolates was $m s r(A)$ (67.5\% versus $43.9 \% ; P=0.011)$. None of HLMUPR-MRSA isolates showed tetracycline resistance. Three predominant resistance gene profiles were found in HLMUPR-MRSA isolates compared with mupirocinsusceptible MRSA isolates. These profiles carried one or two aminoglycoside resistance genes with or without the macrolide resistance $m s r(\mathrm{~A})$ gene. Profile $1: \operatorname{msr}(\mathrm{A})+\operatorname{aac}\left(6^{\prime}\right)$-Ie- $\operatorname{aph}\left(2^{\prime \prime}\right)$-Ia $+\operatorname{ant}\left(4^{\prime}\right)$-Ia (30.0\% versus $0.9 \% ; P<0.001)$, Profile 2 : $\operatorname{aac}\left(6^{\prime}\right)$-Ie-aph $\left(2^{\prime \prime}\right)$-la + $\operatorname{ant}\left(4^{\prime}\right)$-Ia $(25.0 \%$ versus $0 \% ; P<0.001)$ and Profile $3: \operatorname{msr}(\mathrm{A})+$ $\operatorname{aac}\left(6^{\prime}\right)-\mathrm{Ie}-\operatorname{aph}\left(2^{\prime \prime}\right)-\mathrm{Ia}+\operatorname{ant}\left(4^{\prime}\right)-\mathrm{Ia}+\operatorname{aph}\left(3^{\prime}\right)$-IIIa $(12.5 \%$ versus $0 \%$; $P=0.001)$. However, mupirocin-susceptible MRSA isolates were more related to isolates harboring the combination of $m s r(\mathrm{~A})+$ ant (4')-la genes, only the ant(4')-la gene or the susceptible phenotype.

All high-level resistant isolates carried the ileS2 gene. No association between multidrug resistance and HLMUPR-MRSA were found as described by Pérez-Roth et al., (Pérez-Roth et al., 2013). These isolates showed higher gentamicin and tobramycin resistance rates than mupirocin-susceptible MRSA isolates what could be related to the $\operatorname{aac}\left(6^{\prime}\right)$-Ie-aph (2")-Ia presence. This fact has been previously demonstrated with the detection of the $\operatorname{aac}\left(6^{\prime}\right)$-Ie- $a p h\left(2^{\prime \prime}\right)$-Ia and ileS2 genes co-located on the same plasmid (McDougal et al., 2010). 


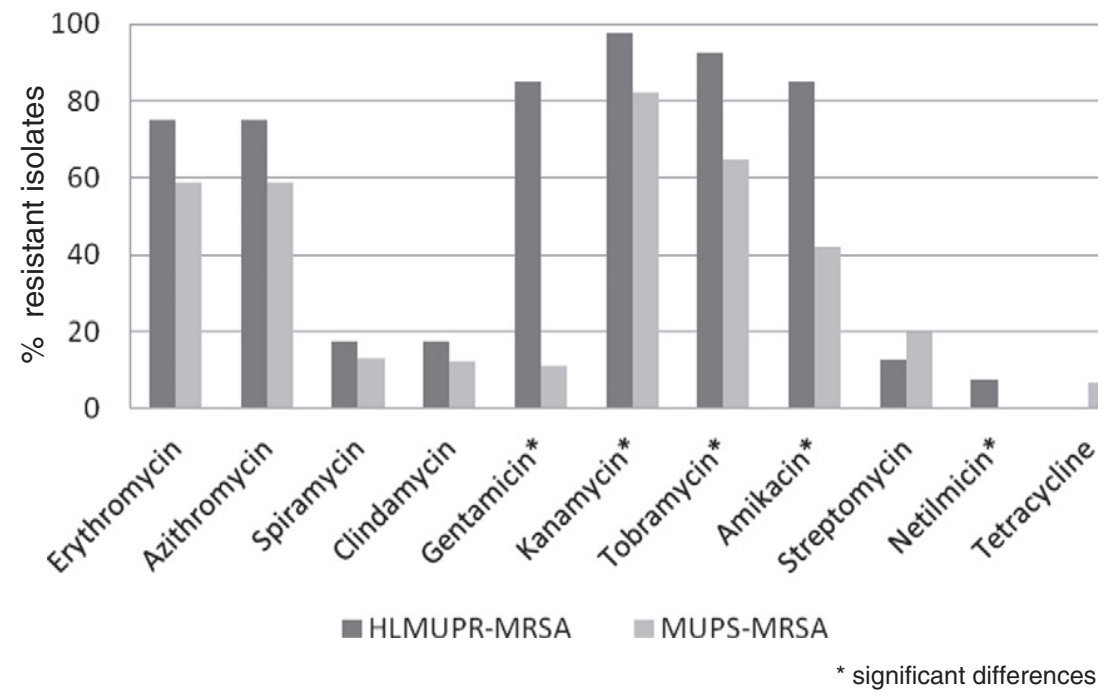

Fig. 1. Percentages of antibiotic resistance detected in high level mupirocin-resistant MRSA (HLMUPR-MRSA) and mupirocin-susceptible MRSA (MUPS-MRSA).

Four different spa types (t002, t067, t2220 and t2226) were observed among HLMUPR-MRSA isolates. The spa type t067 was the predominant one (82\%), although this spa type was also frequent in mupirocin-susceptible group (52\%) where 22 different spa types were found. spa type t067 is the most frequent spa type identified in Spain (Argudín et al., 2009; González-Domínguez et al., 2012, 2015; Lozano

Table 1

Characteristics of 40 HLMUPR-MRSA isolates.

\begin{tabular}{|c|c|c|c|c|c|c|c|c|}
\hline $\begin{array}{l}\text { IS257-ileS2 } \\
\text { amplification } \\
\text { patterns }\end{array}$ & $\begin{array}{l}\text { PFGE } \\
\text { profile }\end{array}$ & ST & spa type & SCCmec & agr & Antibiotic resistance genes & Date of culture & Onset \\
\hline p1 & $\mathrm{C}$ & 125 & t067 & IVc & II & $m s r(\mathrm{~A})+\operatorname{erm}(\mathrm{C})+\operatorname{aac}\left(6^{\prime}\right)-\mathrm{Ie}-a p h\left(2^{\prime \prime}\right)-\mathrm{Ia}+\operatorname{ant}\left(4^{\prime}\right)-\mathrm{Ia}+$ ileS2 & $24 / 07 / 2009$ & Hospital \\
\hline \multirow[t]{5}{*}{ p2 } & $\mathrm{A}_{4}$ & & $\mathrm{t} 2226$ & IVc & II & $\operatorname{aac}\left(6^{\prime}\right)-\mathrm{Ie}-\operatorname{aph}\left(2^{\prime \prime}\right)-\mathrm{Ia}+\operatorname{ant}\left(4^{\prime}\right)-\mathrm{Ia}+$ ileS2 & $28 / 07 / 2009$ & Hospital \\
\hline & $\mathrm{A}_{5}$ & & $\mathrm{t} 2226$ & IVc & II & $\operatorname{aac}\left(6^{\prime}\right)-\mathrm{Ie}-a p h\left(2^{\prime \prime}\right)-\mathrm{Ia}+\operatorname{ant}\left(4^{\prime}\right)-\mathrm{Ia}+$ ileS2 & $10 / 11 / 2009$ & Hospital \\
\hline & $\mathrm{A}_{5}$ & & $\mathrm{t} 2226$ & IVc & II & $\operatorname{aac}\left(6^{\prime}\right)-\mathrm{Ie}-a p h\left(2^{\prime \prime}\right)-\mathrm{Ia}+\operatorname{ant}\left(4^{\prime}\right)-\mathrm{Ia}+$ ileS2 & $24 / 12 / 2009$ & Hospital \\
\hline & $\mathrm{A}_{6}$ & & t067 & IVc & II & $\operatorname{aac}\left(6^{\prime}\right)-\mathrm{Ie}-a p h\left(2^{\prime \prime}\right)-\mathrm{Ia}+\operatorname{ant}\left(4^{\prime}\right)-\mathrm{Ia}+$ ileS2 & $25 / 01 / 2010$ & Community \\
\hline & $A_{10}$ & & $\mathrm{t} 2226$ & IVc & II & $m s r(\mathrm{~A})+\operatorname{aac}\left(6^{\prime}\right)-\mathrm{Ie}-a p h\left(2^{\prime \prime}\right)-\mathrm{Ia}+\operatorname{ant}\left(4^{\prime}\right)-\mathrm{Ia}+$ ileS2 & $26 / 09 / 2009$ & Hospital \\
\hline \multirow[t]{26}{*}{ p3 } & $\mathrm{A}_{2}$ & & t067 & IVc & II & $m s r(\mathrm{~A})+\operatorname{aac}\left(6^{\prime}\right)-\mathrm{Ie}-\operatorname{aph}\left(2^{\prime \prime}\right)-\mathrm{Ia}+\operatorname{ant}\left(4^{\prime}\right)-\mathrm{Ia}+i l e S 2$ & $31 / 05 / 2010$ & Hospital \\
\hline & $\mathrm{A}_{3}$ & & t067 & IVc & II & $\operatorname{erm}(\mathrm{C})+\operatorname{ant}\left(4^{\prime}\right)-\mathrm{Ia}+$ iles 2 & $15 / 04 / 2010$ & Community \\
\hline & $A_{8}$ & & t067 & IVc & II & $m s r(\mathrm{~A})+\operatorname{aac}\left(6^{\prime}\right)-\mathrm{Ie}-a p h\left(2^{\prime \prime}\right)-\mathrm{Ia}+\operatorname{ant}\left(4^{\prime}\right)-\mathrm{Ia}+i l e S 2$ & 29/07/2009 & Hospital \\
\hline & $\mathrm{A}_{8}$ & & t067 & IVc & II & $m s r(\mathrm{~A})+\operatorname{erm}(\mathrm{C})+\operatorname{aac}\left(6^{\prime}\right)-\mathrm{Ie}-a p h\left(2^{\prime \prime}\right)-\mathrm{Ia}+\operatorname{ant}\left(4^{\prime}\right)-\mathrm{Ia}+\operatorname{aph}\left(3^{\prime}\right)-\mathrm{III} \mathrm{a}+$ ileS2 & $30 / 07 / 2009$ & Hospital \\
\hline & $A_{9}$ & & t067 & IVc & II & $m s r(\mathrm{~A})+\operatorname{aac}\left(6^{\prime}\right)-\mathrm{Ie}-\operatorname{aph}\left(2^{\prime \prime}\right)-\mathrm{Ia}+\operatorname{ant}\left(4^{\prime}\right)-\mathrm{Ia}+$ ileS2 & $25 / 02 / 2010$ & Hospital \\
\hline & $\mathrm{A}_{9}$ & & t067 & IVc & II & $m s r(\mathrm{~A})+\operatorname{aac}\left(6^{\prime}\right)-\mathrm{Ie}-a p h\left(2^{\prime \prime}\right)-\mathrm{Ia}+\operatorname{ant}\left(4^{\prime}\right)-\mathrm{Ia}+i l e S 2$ & $13 / 04 / 2010$ & Hospital \\
\hline & $A_{9}$ & & t067 & IVc & II & $m s r(\mathrm{~A})+\operatorname{aac}\left(6^{\prime}\right)-\mathrm{Ie}-a p h\left(2^{\prime \prime}\right)-\mathrm{Ia}+\operatorname{ant}\left(4^{\prime}\right)-\mathrm{Ia}+$ iles2 & $21 / 05 / 2010$ & Hospital \\
\hline & $A_{9}$ & & t067 & IVc & II & $m s r(\mathrm{~A})+\operatorname{aac}\left(6^{\prime}\right)-\mathrm{Ie}-a p h\left(2^{\prime \prime}\right)-\mathrm{Ia}+\operatorname{ant}\left(4^{\prime}\right)-\mathrm{Ia}+i l e S 2$ & $10 / 06 / 2010$ & Hospital \\
\hline & $\mathrm{A}_{9}$ & & t067 & IVc & II & $m s r(\mathrm{~A})+\operatorname{aac}\left(6^{\prime}\right)-\mathrm{Ie}-a p h\left(2^{\prime \prime}\right)-\mathrm{Ia}+\operatorname{ant}\left(4^{\prime}\right)-\mathrm{Ia}+$ ileS2 & $10 / 06 / 2010$ & Hospital \\
\hline & $A_{9}$ & & t067 & IVc & II & $m s r(\mathrm{~A})+\operatorname{aac}\left(6^{\prime}\right)-\mathrm{Ie}-a p h\left(2^{\prime \prime}\right)-\mathrm{Ia}+\operatorname{ant}\left(4^{\prime}\right)-\mathrm{Ia}+i l e S 2$ & $23 / 06 / 2010$ & Hospital \\
\hline & $A_{9}$ & & t067 & IVc & II & $m s r(\mathrm{~A})+\operatorname{aac}\left(6^{\prime}\right)-\mathrm{Ie}-\operatorname{aph}\left(2^{\prime \prime}\right)-\mathrm{Ia}+\operatorname{ant}\left(4^{\prime}\right)-\mathrm{Ia}+\operatorname{aph}\left(3^{\prime}\right)-\mathrm{III} a+$ ileS2 & $17 / 06 / 2010$ & Hospital \\
\hline & $A_{9}$ & & t067 & IVc & II & $m s r(\mathrm{~A})+\operatorname{erm}(\mathrm{C})+a a c\left(6^{\prime}\right)-\mathrm{Ie}-a p h\left(2^{\prime \prime}\right)-\mathrm{Ia}+\operatorname{ant}\left(4^{\prime}\right)-\mathrm{Ia}+a p h\left(3^{\prime}\right)-\mathrm{III} \mathrm{a}+$ ileS2 & 02/03/2010 & Hospital \\
\hline & $\mathrm{A}_{9}$ & & t067 & IVc & II & $m s r(\mathrm{~A})+\operatorname{erm}(\mathrm{B})+a a c\left(6^{\prime}\right)-\mathrm{Ie}-a p h\left(2^{\prime \prime}\right)-\mathrm{Ia}+\operatorname{ant}\left(4^{\prime}\right)-\mathrm{Ia}+\operatorname{aph}\left(3^{\prime}\right)-\mathrm{III} \mathrm{a}+$ ileS2 & $23 / 06 / 2010$ & Hospital \\
\hline & $A_{9}$ & & t067 & IVc & II & $\operatorname{aac}\left(6^{\prime}\right)-\mathrm{Ie}-\mathrm{aph}\left(2^{\prime \prime}\right)-\mathrm{Ia}+\operatorname{ant}\left(4^{\prime}\right)-\mathrm{Ia}+$ ileS2 & $28 / 06 / 2010$ & Hospital \\
\hline & $\mathrm{A}_{10}$ & & t067 & IVc & II & $m s r(\mathrm{~A})+\operatorname{aac}\left(6^{\prime}\right)-\mathrm{Ie}-a p h\left(2^{\prime \prime}\right)-\mathrm{Ia}+\operatorname{ant}\left(4^{\prime}\right)-\mathrm{Ia}+$ ileS2 & $11 / 05 / 2010$ & Hospital \\
\hline & $A_{11}$ & & t067 & IVc & II & $\operatorname{aac}\left(6^{\prime}\right)-\mathrm{Ie}-a p h\left(2^{\prime \prime}\right)-\mathrm{Ia}+\operatorname{ant}\left(4^{\prime}\right)-\mathrm{Ia}+$ ileS2 & $23 / 06 / 2010$ & Hospital \\
\hline & $\mathrm{B}_{1}$ & & t067 & IVc & II & $m s r(\mathrm{~A})+\operatorname{aac}\left(6^{\prime}\right)-\mathrm{Ie}-\operatorname{aph}\left(2^{\prime \prime}\right)-\mathrm{Ia}+\operatorname{ant}\left(4^{\prime}\right)-\mathrm{Ia}+\operatorname{aph}\left(3^{\prime}\right)-\mathrm{IIIa}+$ ileS2 & $11 / 03 / 2010$ & Hospital \\
\hline & $\mathrm{B}_{1}$ & & t067 & IVc & II & $m s r(\mathrm{~A})+\operatorname{aac}\left(6^{\prime}\right)-\mathrm{Ie}-a p h\left(2^{\prime \prime}\right)-\mathrm{Ia}+\operatorname{aph}\left(3^{\prime}\right)-\mathrm{IIIa}+$ ileS2 & $26 / 09 / 2009$ & Community \\
\hline & $\mathrm{B}_{2}$ & & t067 & IVc & II & $m s r(\mathrm{~A})+\operatorname{aac}\left(6^{\prime}\right)-\mathrm{Ie}-a p h\left(2^{\prime \prime}\right)-\mathrm{Ia}+\operatorname{ant}\left(4^{\prime}\right)-\mathrm{Ia}+\operatorname{aph}\left(3^{\prime}\right)-\mathrm{IIIa}+$ ileS2 & $20 / 05 / 2010$ & Hospital \\
\hline & $\mathrm{B}_{2}$ & & t067 & IVc & II & $m s r(\mathrm{~A})+\operatorname{aac}\left(6^{\prime}\right)-\mathrm{Ie}-a p h\left(2^{\prime \prime}\right)-\mathrm{Ia}+\operatorname{ant}\left(4^{\prime}\right)-\mathrm{Ia}+\operatorname{aph}\left(3^{\prime}\right)-\mathrm{III} \mathrm{a}+$ ileS2 & 08/06/2010 & Community \\
\hline & $\mathrm{B}_{3}$ & & t067 & IVc & II & $m s r(\mathrm{~A})+a a c\left(6^{\prime}\right)-\mathrm{Ie}-a p h\left(2^{\prime \prime}\right)-\mathrm{Ia}+\operatorname{ant}\left(4^{\prime}\right)-\mathrm{Ia}+$ ileS2 & $31 / 05 / 2010$ & Hospital \\
\hline & $\mathrm{B}_{4}$ & & t067 & IVc & II & $m s r(\mathrm{~A})+\operatorname{aac}\left(6^{\prime}\right)-\mathrm{Ie}-\operatorname{aph}\left(2^{\prime \prime}\right)-\mathrm{Ia}+$ iles2 & 05/07/2010 & Hospital \\
\hline & $\mathrm{B}_{4}$ & & t067 & IVc & II & $m s r(\mathrm{~A})+\operatorname{aac}\left(6^{\prime}\right)-\mathrm{Ie}-a p h\left(2^{\prime \prime}\right)-\mathrm{Ia}+\operatorname{ant}\left(4^{\prime}\right)-\mathrm{Ia}+i l e S 2$ & $19 / 04 / 2010$ & Hospital \\
\hline & $\mathrm{B}_{4}$ & & t067 & IVc & II & $m s r(\mathrm{~A})+\operatorname{aac}\left(6^{\prime}\right)-\mathrm{Ie}-\operatorname{aph}\left(2^{\prime \prime}\right)-\mathrm{Ia}+\operatorname{ant}\left(4^{\prime}\right)-\mathrm{Ia}+\operatorname{aph}\left(3^{\prime}\right)-\mathrm{IIIa}+$ ileS2 & $21 / 04 / 2010$ & Hospital \\
\hline & $\mathrm{B}_{6}$ & & t067 & $\mathrm{V}$ & II & $m s r(\mathrm{~A})+\operatorname{ant}\left(4^{\prime}\right)-\mathrm{Ia}+i l e S 2$ & 03/11/2009 & Community \\
\hline & $\mathrm{C}$ & & t067 & IVc & II & $m s r(\mathrm{~A})+\operatorname{erm}(\mathrm{C})+\operatorname{aac}\left(6^{\prime}\right)-\mathrm{Ie}-a p h\left(2^{\prime \prime}\right)-\mathrm{Ia}+$ ileS2 & $20 / 10 / 2009$ & Community \\
\hline p4 & $\mathrm{A}_{7}$ & & t067 & IVc & II & $\operatorname{erm}(\mathrm{C})+\operatorname{ant}\left(4^{\prime}\right)-\mathrm{la}+$ ileS2 & $20 / 01 / 2010$ & Hospital \\
\hline p5 & $\mathrm{B}_{5}$ & & t067 & IVc & II & $\operatorname{aac}\left(6^{\prime}\right)-\mathrm{Ie}-a p h\left(2^{\prime \prime}\right)-\mathrm{Ia}+\operatorname{ant}\left(4^{\prime}\right)-\mathrm{Ia}+$ ileS2 & 03/05/2010 & Hospital \\
\hline \multirow[t]{2}{*}{ p6 } & $A_{11}$ & & $\mathrm{t} 2220$ & IVc & II & $m s r(\mathrm{~A})+\operatorname{aph}\left(3^{\prime}\right)-\mathrm{III}+$ ileS2 & $22 / 06 / 2010$ & Hospital \\
\hline & $\mathrm{A}_{11}$ & & $\mathrm{t} 2220$ & IVc & II & $m s r(\mathrm{~A})+\operatorname{aph}\left(3^{\prime}\right)-\mathrm{IIIa}+$ ileS2 & $25 / 01 / 2010$ & Community \\
\hline p7 & $\mathrm{B}_{1}$ & & t067 & IVc & II & $\operatorname{aac}\left(6^{\prime}\right)-\mathrm{Ie}-a p h\left(2^{\prime \prime}\right)-\mathrm{Ia}+\operatorname{ant}\left(4^{\prime}\right)-\mathrm{Ia}+$ ileS2 & 08/10/2009 & Hospital \\
\hline p8 & $A_{1}$ & 5 & t002 & IVc & II & $\operatorname{erm}(\mathrm{C})+$ iles2 & $25 / 05 / 2010$ & Community \\
\hline \multirow[t]{2}{*}{ p9 } & $A_{6}$ & 125 & t067 & IVc & II & $\operatorname{aac}\left(6^{\prime}\right)-\mathrm{Ie}-a p h\left(2^{\prime \prime}\right)-\mathrm{Ia}+\operatorname{ant}\left(4^{\prime}\right)-\mathrm{Ia}+$ ileS2 & $31 / 05 / 2010$ & Community \\
\hline & $\mathrm{B}_{1}$ & & t067 & IVc & II & $\operatorname{aac}\left(6^{\prime}\right)-\mathrm{Ie}-a p h\left(2^{\prime \prime}\right)-\mathrm{Ia}+\operatorname{ant}\left(4^{\prime}\right)-\mathrm{Ia}+$ ileS2 & $12 / 06 / 2010$ & Community \\
\hline
\end{tabular}

PFGE profile: Capital letters (A, B and C) mean strains with different PFGE profiles. The numbers in subscript (1-11) indicate that those strains show closely related PFGE profiles. 
et al., 2013; Menegotto et al., 2012), so this fact could be the reason for its high prevalence in both groups. Among the HLMUPR-MRSA strains, $97.5 \%$ of them belonged to ST125 and 2.5\% to ST5. Both ST are included in CC5, widely distributed in hospital and community settings in Spain (Argudín et al., 2009; González-Domínguez et al., 2012, 2015; Lozano et al., 2013). An association between MRSA CC5/ST125/t067 and erythromycin resistance (encoded by $m s r(\mathrm{~A}) / m s r(\mathrm{~B})$ genes) has been observed. This relation was previously described by other authors (Daskalaki et al., 2009; González-Domínguez et al., 2015; Lozano et al., 2013; Pérez-Roth et al., 2013). All HLMUPR-MRSA strains carried SCCmec type IVc and agr type II, with the exception of one strain included in SCCmec type V that belonged to ST125. None of the studied virulence genes were identified among HLMUPR-MRSA isolates. Pulsed field gel electrophoresis grouped the HLMUPR-MRSA strains in three different PFGE pulsotypes (Table 1). The most prevalent was pulsotype A that included $65 \%$ of the strains. This pulsotype had 11 subtypes where subtype A9 was the most frequent. Thirty per cent of the strains were included in pulsotype B, and subtype B1 was the most frequent. Finally, $5 \%$ of strains were included in pulsotype $C$. These results showed that the strains were closely related.

Analyzing the HLMUPR-MRSA strains selected from vascular surgery we observed the presence of different clones (genetically unrelated).

Considering clinical, microbiological and molecular data, seventyfive percent of HLMUPR-MRSA strains were classified as healthcareassociated MRSA (HA-MRSA) clones (Table 1). The remaining ones were community-onset MRSA (CO-MRSA) isolates related to HAMRSA clones (CO-HA-MRSA). HLMUPR-MRSA was not observed in community-associated MRSA (CA-MRSA) clones.

In HLMUPR-MRSA isolates, distinct IS257-ileS2 spacer arrangements (characterized by a different location of direct repeated copies of ileS2flanking IS257) allowed us to distinguish 9 IS257-ileS2 amplification patterns, named $\mathrm{p} 1$ to $\mathrm{p} 9$. Transfer of ileS2-encoding plasmids is important in the spread of HLMUPR-MRSA as evidenced by the recovery of distinct plasmid configuration types in the same MRSA clone (PérezRoth et al., 2013). We know that the IS257-ileS2 PCR might have exceptional limitations (Pérez-Roth et al., 2006, 2010, 2011, 2013) but we used this PCR as a first and simple approximation to address the molecular epidemiology of plasmid-mediated HLMUPR-MRSA isolates in our clinical setting. Only plasmid sequencing could definitely clarify whether ileS2 plasmids are the same or not. The $\mathrm{p} 3$ pattern was the most frequent in our hospital and community MRSA isolates (65\%) (Table 1). All of them belonged to the same clonal lineage ST125/t067 widely distributed in hospital and community settings in Spain. This suggests a clonal spread in our environment which could explain the high prevalence of HLMUPR-MRSA during the study period.

\section{Conclusions}

Most of HLMUPR-MRSA isolates that are circulating in our environment belonged to ST125/t067. This specific lineage is predominant in our area and it is associated with resistance to aminoglycosides, and to a lesser extent, to macrolides. The presence of the same IS257-ileS2 amplification pattern p3 in 65\% of HLMUPR-MRSA, all of them ST125/t067, suggests a clonal spread in our hospital and community environment which could explain the high prevalence of HLMUPR-MRSA during the study period. An outbreak situation or an increase in mupirocin consumption was not observed.

\section{Funding}

This work was supported by Departamento de Ciencia, Tecnología and Universidad del Gobierno de Aragón, Spain (Project DGA-FSE/Grupos consolidados, B24-211130). MGD received a grant from the S.E.I.M.C (Sociedad Española de Enfermedades Infecciosas y Microbiología Clínica).

\section{Conflict of interest}

None.

\section{Acknowledgments}

We are grateful to Dr. E. Pérez-Roth for his technical assistance in the rapid typing method for strain characterization based on the heterogeneous IS257-ileS2 spacer regions and Dr. J Pintado for his helping with the GelCompar II ${ }^{\circ}$ software.

\section{References}

Aarestrup FM, Agerso Y, Gerner-Smidt P, Madsen M, Jensen LB. Comparison of antimicrobial resistance phenotypes and resistance genes in Enterococcus faecalis and Enterococcus faecium from humans in the community, broilers, and pigs in Denmark. Diagn Microbiol Infect Dis 2000;37:127-37.

Aktas Z, Aridogan A, Kayacan CB, Aydin D. Resistance to macrolide, lincosamide and streptogramin antibiotics in staphylococci isolated in Istanbul, Turkey. J Microbiol 2007;45:286-90

Argudín MA, Mendoza MC, Méndez FJ, Martín MC, Guerra B, Rodicio MR. Clonal complexes and diversity of exotoxin gene profiles in methicillin-resistant and methicillin-susceptible Staphylococcus aureus isolates from patients in a Spanish hospital. J Clin Microbiol 2009;47:2097-105.

Choi SM, Kim SH, Kim HJ, Lee DG, Choi JH, Yoo JH, et al. Multiplex PCR for the detection of genes encoding aminoglycoside modifying enzymes and methicillin resistance among Staphylococcus species. J Korean Med Sci 2003;18:631-6.

Clinical and Laboratory Standards Institute (CLSI). Performance standards for antimicrobial susceptibility testing. Twenty-Fourth Informational SupplementCLSI document M100-S24; 2014.

Cuevas O, Cercenado E, Goyanes MJ, Vindel A, Trincado P, Boquete T, et al. Staphylococcus spp. in Spain: present situation and evolution of antimicrobial resistance (1986-2006). Enferm Infecc Microbiol Clin 2008;26:269-77.

Daskalaki M, Otero JR, Chaves F. Molecular characterization of resistance to mupirocin in methicillin-resistant Staphylococcus aureus isolates in a tertiary hospital in Spain. J Antimicrob Chemother 2009;63:826-8.

González-Domínguez M, Seral C, Sáenz Y, Salvo S, Gude MJ, Porres-Osante N, et al. Epidemiological features, resistance genes, and clones among community-onset methicillin-resistant Staphylococcus aureus (CO-MRSA) isolates detected in northern Spain. Int J Med Microbiol 2012;302:320-6.

González-Domínguez M, Seral C, Potel C, Constenla L, Algarate S, Gude MJ, et al. Antimicrobial resistance, virulence factors and genetic lineages of hospital-onset methicillin-resistant Staphylococcus aureus isolates detected in a hospital in Zaragoza. Enferm Infecc Microbiol Clin 2015;33:590-6.

Jarraud S, Mougel C, Thioulouse J, Lina G, Meugnier H, Forey F, et al. Relationships between Staphylococcus aureus genetic background, virulence factors, agr groups (alleles), and human disease. Infect Immun 2002;70:631-41.

Larsen AR, Stegger M, Sorum M. spa typing directly from a mecA, spa and pvl multiplex PCR assay-a cost-effective improvement for methicillin-resistant Staphylococcus aureus surveillance. Clin Microbiol Infect 2008;14:611-4.

Lee AS, Macedo-Vinas M, Francois P, Renzi G, Vernaz N, Schrenzel J, et al. Trends in mupirocin resistance in meticillin-resistant Staphylococcus aureus and mupirocin consumption at a tertiary care hospital. J Hosp Infect 2011:77:360-2.

Leski TA, Gniadkowski M, Skoczynska A, Stefaniuk E, Trzcinski K, Hryniewicz W. Outbreak of mupirocin-resistant staphylococci in a hospital in Warsaw, Poland, due to plasmid transmission and clonal spread of several strains. J Clin Microbiol 1999;37:2781-8.

Lina G, Quaglia A, Reverdy ME, Leclercq R, Vandenesch F, Etienne J. Distribution of genes encoding resistance to macrolides, lincosamides, and streptogramins among staphylococci. Antimicrob Agents Chemother 1999;43:1062-6.

Lozano C, Porres-Osante N, Crettaz J, Rojo-Bezares B, Benito D, Olarte I, et al. Changes in genetic lineages, resistance, and virulence in clinical methicillin-resistant Staphylococcus aureus in a Spanish hospital. J Infect Chemother 2013;19:233-42.

McDougal LK, Fosheim GE, Nicholson A, Bulens SN, Limbago BM, Shearer JE, et al. Emergence of resistance among USA300 methicillin-resistant Staphylococcus aureus isolates causing invasive disease in the United States. Antimicrob Agents Chemother 2010;54:3804-11.

Menegotto F, González-Cabrero S, Lorenzo B, Cubero A, Cuervo W, Gutiérrez MP, et al. Molecular epidemiology of methicillin-resistant Staphylococcus aureus in a Spanish hospital over a 4-year period: clonal replacement, decreased antimicrobial resistance, and identification of community-acquired and livestock-associated clones. Diagn Microbiol Infect Dis 2012;74:332-7.

Pérez-Roth E, López-Aguilar C, Alcoba-Flórez J, Méndez-Álvarez S. High-level mupirocin resistance within methicillin-resistant Staphylococcus aureus pandemic lineages Antimicrob Agents Chemother 2006;50:3207-11.

Pérez-Roth E, Kwong SM, Alcoba-Florez J, Firth N, Méndez-Álvarez S. Complete nucleotide sequence and comparative analysis of pPR9, a 41.7-kilobase conjugative staphylococcal multiresistance plasmid conferring high-level mupirocin resistance. Antimicrob Agents Chemother 2010;54:2252-7.

Pérez-Roth E, Armas-González E, Alcoba-Florez J, Méndez-Álvarez S. PCR-based amplification of heterogeneous IS257-ileS2 junctions for molecular monitoring of high-level mupirocin resistance in staphylococci. J Antimicrob Chemother 2011;66:471-5. 
Pérez-Roth E, Potel-Alvarellos C, Espartero X, Constela-Caramés L, Méndez-Álvarez S, Álvarez-Fernández M. Molecular epidemiology of plasmid-mediated high-level mupirocin resistance in methicillin-resistant Staphylococcus aureus in four Spanish health care settings. Int J Med Microbiol 2013;303:201-4.

Rodríguez-Baño J, Bischofberger C, Álvarez-Lerma F, Asensio A, Delgado T, García-Arcal D, et al. Surveillance and control of methicillin-resistant Staphylococcus aureus in Spanish hospitals. A GEIH-SEIMC and SEMPSPH consensus document. Enferm Infecc Microbiol Clin 2008;26:285-98.

Schmitz FJ, Jones ME. Antibiotics for treatment of infections caused by MRSA and elimination of MRSA carriage. What are the choices? Int J Antimicrob Agents 1997;9:1-19.

Simor AE, Stuart TL, Louie L, Watt C, Ofner-Agostini M, Gravel D, et al. Mupirocin-resistant, methicillin-resistant Staphylococcus aureus strains in Canadian hospitals. Antimicrob Agents Chemother 2007;51:3880-6.
Sutherland R, Boon RJ, Griffin KE, Masters PJ, Slocombe B, White AR. Antibacterial activity of mupirocin (pseudomonic acid), a new antibiotic for topical use. Antimicrob Agents Chemother 1985;27:495-8.

Udo EE, Jacob LE, Mathew B. Genetic analysis of methicillin-resistant Staphylococcus aureus expressing high- and low-level mupirocin resistance. J Med Microbiol 2001;50: 909-15.

Woodford N, Watson AP, Patel S, Jevon M, Waghorn DJ, Cookson BD. Heterogeneous location of the mupA high-level mupirocin resistance gene in Staphylococcus aureus. J Med Microbiol 1998;47:829-35.

Yanagisawa T, Lee JT, Wu HC, Kawakami M. Relationship of protein structure of isoleucyltRNA synthetase with pseudomonic acid resistance of Escherichia coli. A proposed mode of action of pseudomonic acid as an inhibitor of isoleucyl-tRNA synthetase. J Biol Chem 1994;269:24304-9. 